\title{
How Long is Now? A New Perspective on the Specious Present
}

\author{
Andrea Roselli \\ Durham University \\ DOI: $10.2478 /$ disp-2018-0009 \\ BIBLID [0873-626X (2018) 49; pp.119-140]
}

\begin{abstract}
What is the Specious Present? Which is its duration? And why, ultimately, do we need it to figure in our phenomenological account of temporal perception? In this paper, after introducing the role of the Specious Present in the main models that account for our phenomenological present, and after considering the deflationary objection by Dennett (that the debate relies on the fallacy of the Cartesian Theatre of Mind, the idea that it is meaningful to ask where and when an experience becomes conscious), I claim - thanks to a spatial analogy — that there could be a good criterion to distinguish between a present experience and a past experience, that there are good reasons to sustain the Specious Present (while snapshots are in no sense part of our phenomenological life), and that there could be a precise way to define the nature - and to measure the duration — of the Specious Present; as I will clarify, our capability and possibility to act and react are central in this perspective. If we accept this change of perspective, there is a definite sense in which the Specious Present is part of our temporal phenomenology.
\end{abstract}

\section{Keywords}

Philosophy of time, temporal experience, specious present, coconsciousness, experiential now.

\section{Introduction}

Thomas Reid, underlining the contradiction between strict philosophical truths and common sense, claimed that in our ordinary life we allow 'the present' to indicate a duration, rather than - as it should be - a point-like moment. In any duration there is a before and an after; strictly speaking, then, the present is durationlessotherwise it would contain some past elements and some future ele- 
ments. However, this line of reasoning was not able, in the opinion of the American philosopher and psychologist William James, to capture our immediate experience of change, persistence and motion. James wanted to differentiate what he meant by 'present' from this objective, mathematical description of a point on the temporal line. Absorbing the results of the experimental psychology done in Germany in the second half of the $19^{\text {th }}$ Century, and framing it in terms of a philosophical idea that he himself credited to E. R. Clay, James made very famous the claim that the phenomenological now is not point-like, but extends over an interval of time. He had in mind a phenomenological present, a duration which is perceived both as present and as temporally extended. This phenomenological present is 'specious' in that, unlike the objective present Reid had in mind, it is an interval and not a durationless instant. There is a sense, then, in which all the contents of a Specious Present possess a sort of phenomenal presence; the aim of the present paper is to better specify this sense.

\section{Which phenomenological present?}

There is a preliminary, methodological issue: we must specify which phenomenological present we are referring to- the period of time to which we have a vivid cognitive access, or the (much shorter) window through which we are directly aware of change? In the relevant literature we find a lot of estimated different durations for the Specious Present, from 300milliseconds to 12seconds; such an incredible difference could be explained only by admitting that not everyone is speaking of the same thing. James for example, although mentioning the time lapse necessary to hear two auditory stimuli in succession, chose to characterize the Specious Present as "the maximal extent of our immediate distinct consciousness for successive impressions" (James 1890: 612) —which is approximately 12 seconds, as he learned from the German physician, physiologist and experimental psychologist Wilhelm Wundt. Wundt's experiments were meant to measure the capability to recall accurately an auditory sequence, something which is manifestly different from the direct experience of motion. James, then, thought of the Specious Present as the period of time to which we have a vivid cognitive access, while in the second part of the $20^{\text {th }}$ Century a narrower meaning started 
to settle, attesting the duration of the Specious Present around 2/3 seconds.

One of the most influential figures, in this sense, is Ernst Pöppel. Studying the reproduction of visual and auditory stimuli of different durations, Pöppel observed a mechanism which appeared to integrate successive events within a temporal window of approximately 2 to 3 seconds; subjects typically mentally structure auditory sequences, experiencing successive beats as a unit; but "it turns out that two beats cannot lie further apart than 2 to 3 seconds to allow subjective accentuation. Beyond this interval, it is no longer possible to mentally connect the second to the first beat, i.e., the first beat has then already disappeared into a perceptually not directly available past [...]. It is as if the brain asks, 'What is new in the world?' every 3 seconds or so" (Pöppel 1997: 114); analogously Elzbieta Szelag, analyzing a series of experiments in which sequences of metronome beats at different frequencies have been presented to different subjects, commented: "the results indicate an integration process, i.e., temporally separated successive beats are mentally connected with each other into larger perceptual units [...]. It appears that the sensory systems can hold information up to the temporal limit of approximately 3s" (Szelag 1997: 122). While, however, everyone admits that these results are meaningful - that $2 / 3$ seconds cycles are relevant to our brain processes - nowadays most of the philosophers of time tend to think that a much shorter figure is a more plausible candidate for the extended feeling of presentness (while in three seconds we typically lose the feeling of direct awareness ${ }^{1}$ ).

Such authors (among others: Lockwood 2005, Le Poidevin 2007, Strawson 2009, Hoerl 2014, Prosser 2016, Dainton 2017), interpret the Specious Present as the maximum duration in which change or succession can be experienced as a whole, object of a single mental act. If we take the Specious Present to be the window through which we are directly aware of change and persistence, then it is plausible to suppose that it is of the order of about a second. Even between the authors of this 'third generation' of the Specious Present, however,

${ }^{1}$ Dainton (2017: 7.1): “Clap your hands three times, leaving about a second between each clap; when the third clap takes place, are you still directly aware of the first?" 
there are differences ${ }^{2}$ in the estimates of the duration of it. What they are thinking of is the phenomenological immediacy associated with our complex perceptions; I am proposing here a model capable of establishing a definite meaning for this 'phenomenological immediacy' and of avoiding the 'Cartesian theatre of the mind' fallacy. Before doing that, however, it is crucial to present the main models of our temporal phenomenology and to show how the Specious Present appears in them. What I mean to show is that every model has a story to tell about our phenomenological immediacy and that - if we reject the change of perspective I propose here - we don't have a way to choose. The three main accounts of our experience of time and presentness are the Cinematic Model, the Retentional Model and the Extentional Model.

\section{Three models of our temporal phenomenology... plus one}

Cinematists reject the idea of a Specious Present. They maintain that our temporal phenomenology is a succession of momentary states of consciousness. We always know what comes first and what second; therefore, the best model to describe our temporal awareness is one in which there are momentary states of consciousness (with momentary I mean physiologically momentary: about $30 \mathrm{~ms}$, time under which we can't distinguish the order of two stimuli ${ }^{3}$ ). Of course, it can be argued that our present experience is in fact momentary, but there are some reasons that explain why it doesn't seem so (we remember the immediate past, or there is a retention of the immediate past in the present experience, etc.). Something that is usually contested by the opponents of the Cinematic model is that having in mind the different positions that an object occupied in time, and having the cognitive understanding that it moved, does not coincide

${ }^{2}$ Dainton (2000), for example, opts for half a second or less; Lockwood suggests a second or a second and a half (2005). Strawson goes the other way, and suggests a figure of around $300 \mathrm{msec}$ (2009): there is not a widespread consense.

${ }^{3}$ Stimuli of around $1 \mathrm{~ms}$ need to be separated from one another by in interval of around $30 \mathrm{msec}$ if they are to be perceived as a succession - a result which holds across sensory modalities. Stimuli which are separated by shorter intervals are not perceived as distinct. 
with directly perceiving it moving. ${ }^{4}$

It is mainly to save this last intuition that the two other models of temporal experience were born. Retentionalism and Extentionalism are realists about phenomenal temporality — change, succession and persistence can be directly perceived or apprehended. ${ }^{5}$ Both Extensional and Retentional theorists agree that a temporal spread of contents can be apprehended as a unity. Not only, then, simultaneous contents can be experienced together, but even contents that are successive; contents which are apprehended as unified in this way belong to a single Specious Present. How is it possible, however, to perceive an extended present? When we hear three close auditory tones, even if we experience the whole musical phrase as present, yet we also hear the notes as successive, and therefore as extending over an interval. How could a succession of elements also be experienced as present? Retentionalist and Extentionalist, while accepting both the idea of a Specious Present, give different accounts of this apparent paradox.

Retentionalists agree that our experiences occur within episodes of consciousness which lack an objective, clock-time extension, ${ }^{6}$ but these episodes, they maintain, are composed by an immediate experience and a representation (or retention) of the recent past; the result is that the contents of these experiences represent temporally extended intervals. The stream of consciousness, then, is composed of succession of momentary states - just as the Cinematists claim; the difference is that the experience of these momentary states is one of duration. The confinement to a momentary present is seen by Retentionalists as a condition for contents to be experienced together; phenomenal unity needs the simultaneous presentation of contents

${ }^{4}$ Obviously enough, many refined arguments have been put forward by Cinematists to defend their position. All I'm trying to do here, however, is to present the main models of our temporal phenomenology to show how the Specious Present is present in them.

${ }^{5}$ There is the possibility to build a 'Cinematist Realist' model, but virtually every philosopher of time who defends Cinematism is an Anti-realist about phenomenal temporality.

${ }^{6}$ There obviously are some border-line accounts (see for example Lee 2014), but I am here presenting the position in the most general and neutral way possible. 
to a single momentary awareness. James himself - in the Principles, at least - seems to subscribe this way of thinking of the Specious Present: a temporal window which does not extend over an interval of objective time; Retentionalist's Specious Present possesses only an experienced extension. A distinction is usually drawn between the content and the vehicle of a representation: the former is that which is being represented, the latter is the entity which carries the representation; experiences can represent temporal features, but they also themselves possess temporal features. ${ }^{7}$ The temporal properties of the objects we perceive, in fact, need not coincide with the temporal properties of the presentations (episodes of awareness) in which we apprehend them; the properties of a representation (that which is doing the representing) and the content of that representation (what is represented) can differ dramatically. Objects which are objectively past can be presented in our present experience; there is no obvious reason why the temporal properties of a mental representation need to entirely coincide with the temporal properties of the content carried by it.

Retentionalists, however, are sometimes accused to have invented "nothing but a new word", as Dainton (2000: 155) puts it: what is a retention, and in what differs from a memory? The standard answer is that we can think of retentions as past-directed mental representations, automatically associated with every experience; unlike memories, they do not unfold over time, and they are more vivid than ordinary memories. Modal Retentionalists, such as Husserl and Brentano (at least in his later writings), argue that there are temporal modes of consciousness - objects can be apprehended as past to differing degrees; contents appear under different 'temporal modes of presentation', some fully-present, other 'past', other 'more past', and so on. Non-Modal Retentionalists, on the other hand, maintain that all the

${ }^{7}$ Divergences about the content / vehicle distinction lie at the heart of the debate between Extensionalists and Retentionalists. Retentionalists believe that experiences which present us with succession are able to disguise their true temporal properties: a succession of presentations, they claim, can amount to a presentation of succession only if the contents are presented to a single, momentary act of apprehension. The Extensional approach carries no such implications: on this view, a Specious Present present is itself temporally extended. Here vehicle and content have the same temporal properties. 
contents within a Specious Present appear equally present - the only difference with Extentionalists being the relationship with clocktime. Both versions encounter some possible objections: Non-Modal Retentionalists lack a convincing way to describe the uniqueness and distinctiveness of our experiences, it seems we should hear the same notes many times; Modal Retentionalists, on the other hand, have the difficult task to explain, if retentions are unlike immediate experiences, how and why we have direct perceptions of change and motion - the experience of change would be somehow different from that of a shape or a colour. ${ }^{8}$

Extentionalists claim that the Specious Present is not merely experiential, but extends over clock-time. They hold that the atomic unit of our perception is an extended period of time; we have an experience of succession because we directly experience the succession. The Retentionalist doctrine that diachronic phenomenal unity can only exist in strictly momentary states of consciousness is rejected, in favour of a more 'natural' model of temporal awareness. Change and persistence are incorporated in our experience in a quite straightforward way, since our stream of consciousness is composed of a succession of extended chunks of experience. The Extentionalists' Specious Present is itself temporally extended, and its parts succeed one another in time in just the way they seem to; our experiences extend over a period of real time, in a way which (almost infallibly) matches the phenomenal period it presents.

Finally, in the last years another anti-realist alternative to the 'Specious Present' has been put forward. Rather than straightforwardly denying that we directly experience motion, the antirealist stance is made more plausible by a credible explanation as to why we believe we experience motion (and more generally, change) if in fact we do not. This improved version of the Cinematic model, suggested in particular by Prosser (2016), is the Dynamic Snapshot Theory. In particular, it is claimed that motion can be directly experienced despite the unextendedness of the atomic content of our experience. It is true that experience consists of a series of perceptual snapshots, one after the other (note that this could be a continuous

${ }^{8}$ Again: there are many good arguments in defence of these claims, but it is not necessary to mention them here. 
series - we don't have to imagine experience as fragmented into countable snapshots); these snapshots, however, are not bound to be static, since even if experience has an instantaneous content, it isn't true - it is argued - that this cannot include something that can be detected over a non-instantaneous interval. The Dynamic Snapshot Theory posits a vector-like feature that durationless contents could possess; even an instantaneous experience could contain vector rates of change, being then an immobile experience of something moving. Even if it is technically true that the atomic content of our experience (again: around 30ms, the threshold for a definite experience ${ }^{9}$ ) is a snapshot, that doesn't mean that that content has to be static. Extended processes, then, have instantaneous parts, even if the nature of such parts depends on what occurs at other time. Consider Prosser (2017):

'Moving' is a state that something can be in at an instant, even though it can only be in that state by virtue of being in other places at other times. (Prosser 2017: 150)

It would, of course, take time for the brain to detect motion, for example by comparing patterns of retinal stimulation at different times. But it clearly does not follow from this that the resulting experience must have a temporally extended content. The necessary properties of the stimulus can differ from those of the resulting experience. (Prosser 2017: 153)

When we have an experience of motion, we are in a state that has a representational content that determines the character of the experience: put it simply, when we have a visual experience of a car going at 50mph, we don't only see different photographs (static snapshots); the acquisition of the last snapshots define an experience of motion, so that in every snapshot we see, so to speak, a photograph of a car moving; our memory integrates the visual experience with the crucial vector of motion. Even in this model, then, for there to be a 'dynamic snapshot' there must be some kind of retention of information; something must tell us that motion is painted on the present snapshot.

\footnotetext{
${ }^{9}$ See for example Wittmann (2011). Technically, then, even our point-like experience is extended, but we can understand in what sense it is a snapshot: it is like a photography in which the shutter remains open for $30 \mathrm{~ms}$; everything that happens in that window of time is simultaneous for us.
} 
At this point, one could start doubting that there is a genuine debate between the models. How is our phenomenological introspection supposed to give support to one or the other model if every model have the same capability to distinguish between different kind of experiences - simply, with different names? I agree with Prosser (2016: 136): "it is not really clear that we must choose between a theory that combines instantaneous contents with a short-term memory and a theory according to which there is a short-lived Specious Present". Will we be ever able to understand if our visual experience of a car going at $50 \mathrm{mph}$ results either from a comparison between the last snapshot and the preceding snapshots (short-term memory), or from an extended experience, or from a retention of the past experiences? But even more radically: is there a difference at all?

It seems that all the models tell the same story about information processing; some preceding data must be combined with the last acquired data in order to produce our experience of motion. Of course, every model has a specific line of defense, but it doesn't seem that any of them contain elements capable of explaining phenomenological features that the other models can't; maybe they simply tell the same story with different names. As I am going to argue in the next section, the fallacy of the Cartesian Theatre model of the human mind has a central role in this line of reasoning. If this is the case, the Specious Present would merely be another name to convey an idea, but nothing specific, nothing that we would be able to clearly individuate in our phenomenology. However, I don't think that this is the case, and I am going to offer an argument to believe that it is not.

\section{A difficulty for the phenomenological dispute}

Daniel Dennett (1991) argued that, in some cases at least, there is no sharp dividing line between memory and experience, and the project of trying to ascertain the temporal microstructure of consciousness is misconceived. If two stimuli are presented in rapid succession, for example, most subjects will be able to identify the second stimulus far more reliably than the first. The standard interpretation of this sort of experiment, according to Dennett, is that in such cases the subject do not experience the first stimulus; they have no visual experience of it, since the occurrence of the second stimulus somehow 
interferes with the normal perceptual process. However, there is a second possible interpretation of the subject's responses. Perhaps the subjects do experience the first stimulus, but something interferes with their memory of this experience, and so when subsequently queried they deny having seen it. ${ }^{10}$ Dennett maintains that in the case described (and others like it) neither interpretation is correct; there is simply no fact of the matter as to whether or not the subject's experiences the first stimulus: "the boundary between perception and memory $[\ldots]$ is not perfectly sharp" (Dennett and Kinsbourne 1992: 192). The assumption that there must be a determinate answer is grounded, in his opinion, in a sort of Cartesian conception of experience; according to such a conception, the question 'what is currently appearing on the stage of your consciousness?' always has a fully precise answer, an answer determined by the experiential contents present in the relevant subject's consciousness. Dennett's arguments, it has been claimed, ${ }^{11}$ have a 'verificationist slant', relying as they do on the principle that if there isn't evidence that $\mathrm{P}$ obtains or not, there is not fact of the matter as to whether or not P obtains. In my opinion, however, the argument is - more radically - that there simply is not a point at which external data become a conscious experience, or a conscious experience becomes a memory; it is not only difficult to discover - there is not such a region in our brain.

Inspired by Dennett's argument, Simon Prosser (2016) argued that the differences between the models of our temporal experience seem to concern the point at which external data become conscious ${ }^{12}$ - where to put the line between memory and present consciousness. If, for example, it is a temporal extension of $500 \mathrm{~ms}$ that, all

${ }^{10} \mathrm{He}$ calls the first mechanism Stalinesque, in that the experiences our perceptual systems produce do not accurately reflect the objective facts - in a manner reminiscent of Stalin's show-trials, and the second mechanism Orwellian, in that a false version of recent events is being rewritten, in a manner reminiscent of Orwell's dystopian societies.

${ }^{11}$ See for example Dainton 2017.

${ }^{12}$ This is Dennett's position, not mine: I think that the debate between the different models is genuine; simply, there are many ways to formulate it. I present Dennett's objection, however, because it gives an interesting insight in what I have to say in the following. 
together, is presented by our ocular nerves to our brain — let me say consciousness - so that we get to know the motion happened in front of us in the last $500 \mathrm{~ms}$, we have a direct experience of a temporal extended atom - and Extentionalists and Retentionalists are right; if, on the other hand, what happens is that different atoms - say, snapshots taken every $30 \mathrm{~ms}$ - are presented in succession to our brain, we have a direct experience of a snapshot taken in the last $30 \mathrm{~ms}$ by our eyes, and a short-term memory of the preceding snapshots - and Cinematists are right. Such a debate, Prosser claims, seems to presuppose the fallacy of the Cartesian Theatre of the mind: the idea that there is a place and a moment in which the mere perceptual external data become conscious experience-as if a consciousness homunculus lived inside our head and watched the data presented to him. Besides reasonable worries of an infinite regress, the point is that our conscious experience is much more diversified and complex than this and, most of all, there isn't a finish line — a modern pineal gland, so to speak.

I agree on this with Dennett and Prosser: there isn't any fully conceptualised experience, happening at a definite time, as opposed to the process of obtaining it; it is the process itself that constitutes our experience in its different degrees of consciousness. Our cognition of the external world begins in the eye, in the ears, in the fingers; there is a process of rising consciousness, of course, but it would be vane to look for a precise locus where we come to meet an external phenomenon. It is pointless to try to distinguish between the real consciousness, the real person, the one that knows and understands, and the mere senses and nerves that, like tools and wires, bring information to the person, and to ask ourselves when the real self come to know something, when it is directly perceiving it or only remembering. If the difference between the models, then, consist simply in where the finish line should be placed, the non-existence of a finish line should deflate the whole debate. There is, of course, a phenomenal character associated to our processing of the external input, but - as Prosser (2016: 154) puts it - "we need not think that there is an answer to the question: 'when is it like that for the subject?' [...] finer-grained questions about what the subject was experiencing at some specific time simply have no good answers".

A real, human experience takes time to be formed. Within this 
extension of time, it is not clear at all what is a direct perception and what a short-term memory; it is not even clear if there is, or should be, such a distinction. At which moment a conscious experience ends and become a memory? The mathematical description of the present as a point on the temporal line should not be confused with our phenomenological present, which consists in a cognitive blend of the last apprehended data and - fact that is often underestimated — an anticipation of the future; our understanding of external environment is almost totally focused on our capability to intervene on it, or escape from it - everything, in our spatial and temporal basic observation of the world, is centred on our possibility to act.

This line of thought, however, sheds a new light on the debate about the Specious Present. If the dispute is merely about the point at which a visual information become a conscious experience and then become a memory, there isn't much hope. But if we intended it, instead, as a debate about the experiential 'here-now' — much nearer to our phenomenological and practical life - there is the possibility of a new dawn for it. In this case, the debate would be a genuine dispute about the best model to account for a certain phenomenal intuition, which has even a definite physiological counterpart, as I am going to show in the next section.

\section{The debate in a new vest}

Think of what are our senses, and why we have them; animals are the structured organisms that can move. The evolutionary reason of the functional and integrated role of our eyes, our ears, our nervous system, is to permit us to move in, intervene on or escape from the external environment or other animals. Our cognition of space and time is not unrelated to this logic; it would be an error to think of us as organisms with such and such characteristics, such and such temporal and spatial phenomenology, which are then lowered in a particular world, as Adam and Eve, shaped in Eden and then fallen on Earth. It is the world itself that shaped us and our evolution in it - our understanding of it, and the possibility to act, to move, is a central part of the project.

The mechanism that underlies our capability to grab objects is a perfect example of that. Before the discovery of brain neurons, it 
was natural to think that when we have an object in front of us-20 centimetres or 2 metres away - we can decide to take it or not; if we decide to take it, our brain tells our arm to move and take it — or, if it is too far, tells our body to walk there and our arm to take it. Nowadays, however, we know that what really happens is much more complex; ${ }^{13}$ there are motor neurons firing for every object in our proximity, and an inhibitory mechanism blocking the communication between them and the nerves; the motor neurons firing are not only continuously repeating to the arm how to coordinate to grab the object, they are literally telling it to take it; it is only thanks to the inhibiting role of the motor cortex - region of the cerebral cortex in the frontal lobe - that we don't actually take every object within our reach. Experiments ${ }^{14}$ with the fMRI demonstrated how, if we move the object out of our possibility of reach, the motor neurons stop firing; of course we can still see the object and think 'I want to take it', but it is a completely different cognitive action. The curious fact is that, if we give the person doing the experiment a stick (with which she could reach the object) the neurons start to fire again.

The moral of the story is that our possibility to directly and immediately intervene on the external environment is something that makes a great difference for us, it is the way we are built; we have senses for that reason. The 'here-now', related to our particular possibilities (how long are our arms, if we carry a stick or not, etc.), is central to our way to experience the world. At first glance, we could have thought that there isn't a clear sense in which an object is 'here'; whether it is 20 centimetres or 2 metres far, it is always 'here' in some sense. A debate regarding the exact point at which an object is 'spatially present' for us would have been meaningless; there is not a point at which the object changes its status and becomes present, we could have argued. But we are not Adam and Eve, the 'spatial here' - intended as 'what I can directly and immediately act on', 'what I can reach' - makes a great difference for us, both from a neurophysiological and a phenomenological point of view. There is an extended spatial 'here' clearly distinguished and individuated, $2008 \mathrm{~b}$.

${ }^{13}$ See Rizzolatti et al. 1996, Rizzolatti et al. 2000, Sinigaglia 2008, Sinigaglia

${ }^{14}$ See for example Bear et al. 1996. 
and the debate regarding different models trying to describe the situation would be meaningful. I think that a similar point could be made in the temporal case; before turning to the temporal version of this reasoning, however, let me push the argument a little further.

Think of the famous phi phenomenon (the phenomenon of apparent motion). If two immobile spots of light on a screen are turned on and off at certain moments (generally the interstimulus interval must be around 30 frames per second), we see-instead of the two dots - one dot moving; Dainton's comment is that "evidently our brains are more than happy to supply us with experiences of motion at the least opportunity" (Dainton 2017b: 1); but why? Again, I think that the reason is that we have been built by nature; if we see, in the sky or in a field, a black dot disappearing and very briefly another black dot appearing 30 centimetres at its left, the best explanation of that is that something is moving - and not that the first dot simply vanished in the sky, while another one miraculously came into existence; as Hoerl (2013: 162) puts it, "temporal features of reality can enter into the content of perception in the light of the immediate implications they possess for actions"; a similar point is also made by Morgan (2003: 61): "we are not normally conscious of a blur in moving objects: nor do we see them frozen in space-time. Instead, we see recognisable objects in motion". For the same reason, we see the leaves of the trees of the same green in the morning and in the evening - while, in reality, the two perceived colours are totally different, and mostly not green. All our conscious perceptions contribute to a successful and homogeneous experience of the world we live in; our brain continuously tries to connect every perception to familiar experiences, experiences that it knows how to react to, and this is why it is so easy to artificially create perceptual illusions in a laboratory using vanishing and appearing objects. My point, then, is that we can't think of our experiences without thinking of the way we are built; I agree with Hoerl (2013: 168) when he claims that "in perception [...] features of reality are represented in the light of their immediate relevance for the subject's actions".

My argument, then, is that the possibility to act on particular objects or events is something that makes a great difference for our experience of the external world; just as in the spatial case there is a distinct sense in which the present is the 'reachable here', I believe 
there are good arguments to claim that in the temporal case 'the present' is the extension of time in which we can react to what's happening without the sensation that it is already 'too late'; some thought experiments will help me to clarify this point. All the arguments given before, about the complexity and the variegation of our temporal experience, stand, but there are also good arguments to claim that the dispute could rise again in this new vest.

Consider this example made by Prosser (2017: 154):

Imagine hearing do followed by, say, ten seconds of silence during which you continue to think of what you heard, followed by re [...]. I predict that you will have no difficulty in detecting that the first note was followed by a note approximately one tone higher. [...] Now imagine the sequence do-re played faster, taking only a fraction of a second. Is there any phenomenological difference beyond the fact that the sequence takes less time?

Until we look for a phenomenological difference in terms of memory Vs experience we won't find one, since it is not possible to clearly distinguish these two elements of our cognitive life-since there is not a pure experiential datum being presented to the mind. But if we, differently, set the debate as concerning the now-here of our phenomenological life, it is possible to discern a demarcation, and the result — as I am going to show in the following — will favor the 'extended present accounts' of our temporal phenomenology (Retentionalism and Extentionalism), instead of the 'snapshots ones' (Cinematism or Dynamic Snapshot view); a Specious Present, that is to say, seems to be a part of our temporal phenomenology.

Think of the experiment described by Prosser; his conclusion was that there wasn't any clear phenomenological difference, besides the platitude that one sequence took more time. Imagine that this time the experimenter asks the subject to express a preference, an aesthetic judgement for example, between the notes of a piano- - "pick your favourite note", could be the assignment. In the first case (the note do followed by ten seconds of silence and then a re) the subject has the time to react, to consider how much she likes the note do; some seconds later she hears the note re and considers it, in turn; obviously enough, it is not particularly difficult for her to acknowledge that the second note was higher. But maybe the point is another; 
when she hears the second note, she has already judged the first one, which then feel past. From a phenomenological point of view, there is a clear, distinct sense in which the first note is past - the subject already reacted to it. In the second example envisaged by Prosser, on the contrary (the sequence do-re played faster, taking only a fraction of a second), we don't have the physiological or phenomenological temporal space to 'act' on the first note and then on the second; of course we know that one preceded the other, but in a clear sense we are presented with two notes and we have to make two judgements in one session. We could even have some difficulty in aesthetically judging the two experiences separately (try to do it. You will have the clear phenomenological impression that you are asked to judge two things all at once). It is legitimate to have different intuitions on that, and maybe I represent an exception, but it seems to me that there is a very definite sense in which the two notes played in a fraction of a second feel equally present and in which the two notes played ten seconds apart don't.

I take it that William James had in mind something along these lines when he spoke, in the Principles, of a 'duration-block':

we do not first feel one end and then feel the other after it, and from the perception of the succession infer an interval of time between, but we seem to feel the interval of time as a whole, with its two ends embedded in it. (James 1890: 610)

In this context it is possible to recover Dainton's intuition of a 'coconscious present', a temporal extension that allow us to have only one reaction. Realizing to having heard two close sounds is something different from realizing to having heard one sound and nine seconds later realizing to having heard another sound, one tone higher. Co-consciousness, then, would be defined by the ratio between the temporal distance of the two sounds and our capability to react (in this case, judge them). The rate would define then an extended now, just as in the spatial case the length of our arms and the distance of the object (which together determine our possibility to reach something) define our extended here.

A new grasp on the concept of co-consciousness would be thus reached. Two very close sounds are co-conscious, in this sense, because we can have only one reaction. We don't have the time to 
notice that there has been a sound, and then another one; we have an experience of two close sounds. When there are many close sounds going on for many seconds, instead - for example, think of a piano song - what we do is finding a rhythm of many extended presents. Of course we can generate cases in which it is difficult to distinguish between one experience of two sounds or two distinct experiences, but I don't see it as a troublesome problem. Most of the times, there is a clear phenomenological sense in which something is present or here in this specified sense; I take it to be a promising approach to understand what the Specious Present is. It is always possible, strictly speaking, to break down an experience and arrive at an atomic snapshot, but this is not what we have in mind when we think of an analysis of our temporal experience; we look for a model that is capable to translate phenomenological differences, instead of merely chronometric. When the Extentionalist claims that our present experience is extended, she has - or should have - in mind a notion of co-consciousness as now described. It is now the Cinematists (Static or Dynamic) that should explain in which sense our experience is point-like.

The question of whether our temporal experience has a temporally extended content should be rejected, rather than answered, only if we think of it as the search of a definite moment at which information 'enters' or 'leaves' consciousness, or at which conscious experience starts and ends; if we, instead, consider the temporal content of our experience as the now with which we have a particular interactive role, just as the spatial here that our motor neurons are so good at individuating, then the question becomes interesting again. We could ask which is the extension of our present temporal experience just as we can ask which is the extension of our reachable here; in this case, some sort of Specious Present seems an indispensable element of our temporal phenomenology. It is only after such a philosophical analysis that a neurophysiologist can define the nature of the Specious Present and measure its duration.

\section{The role of anticipation and a counterargument answered}

Finally, I want to stress the (often neglected) role of anticipation in the picture. The arguments about the phenomenological present 
usually concern our sensations, short-term memory and long-term memory. However, even the anticipation of the near-to-come future has an important role in the definition of our temporal present window; let me make an example, before turning to some experimental data. It is exactly talking of action and reaction that the role of anticipation came to the surface in such a strong way: not only, in fact, we react to something that we have seen, or touched, or smelledthat is gone, happened, in the past- , but we also act according to what we want to do in the future. It is only keeping in mind the combination of these two elements, past and future, reaction and action, that we can understand the window of the present, and not only because they always coexist in that window, but even and more significantly because one influence the other, as some very interesting experimental results confirm. One that I find significant, in this context, and that stresses the role of anticipation in our temporal experience of the present, is the phenomenon described by psychologists as backward masking: when, for example, we listen to music, the phenomenal character of our experience of a note is affected by the properties of the notes immediately prior to that note and after that note. Laurie Paul (2014: 186) comments:

How can we 'see into the future' in this way? What is the basis for this experience of foreshadowing? There is debate about the mechanism involved in the cognitive processing of these temporally clustered events. Some have argued that it is a predictive effect [...]. Others have argued that it is what is called a 'postdictive effect' [...]. What matters here is that these foreshadowing and filling-in psychological effects are empirically well-documented, even if their source is not fully understood.

What I find particularly meaningful, in this context, is that this backward masking effect obtains only when the stimuli are close to one another; I see it as an experimental confirmation of the fact that there is indeed a phenomenological difference in the two sequences of notes 'do-re' beyond the mere fact that one sequence takes less time. When two notes are experienced in the same Specious Present, we have one reaction to two sounds - the second sound could even affect the phenomenal character of the first one, while the same thing does not happen when two sounds are more temporally sepa- 
rated. A similar reasoning can be extended to the other senses. ${ }^{15}$

At this point, however, it is very easy to see a possible counterargument. If we don't specify the length of the specious present, it can be argued, we haven't actually brought about any kind of improvement to the debate. A stronger version of this argument could be that if we don't specify the length of the specious present, we don't even have arguments to maintain its existence, not even from a phenomenological point of view; affirmanti incumbit probatio, it could be said. If we are interested in maintaining the importance of the specious present, we should be prepared to answer the very reasonable worries about its extension. If the specious present is our phenomenological window of presentness, how extended is that window? Is it something that should be decided by a phenomenological investigation, or is it the field of neurophysiological studies? I see the difficulty here, but I do not think it actually is a counterargument to what I am proposing, which is merely a change of perspective. A lot of experimental work should be done on this topic, and something more precise about the extendedness of that window could be said. My point, however, was that the important thing to understand here is that the experiments should not be conducted in the belief that we are going to discover the border between present consciousness and memory; instead of focusing on what particular cognitive acts are going on in our mind, the experimenters should focus on our possibility to act and react. My intuition is that the results (the extendedness of the specious present) will vary depending on the particular task set by the experimenters, but I don't see it as a problem. It is quite natural to think that the window of our phenomenal presence has a different extension depending on what we are doing or trying to do. The important point, in my opinion, is that once we have absorbed this change of perspective there is a definite way to decide if a Specious Present is part of our temporal phenomenology, to understand what it is, to measure what is its duration in a particular situation, and to decide which models of our temporal understanding are more apt to describe our phenomenal temporality. Conclusions In the first section, I have presented James' idea of a present which is specious in

${ }^{15}$ See for example Saccuzzo et al. 1996, Herzog et al. 2013 for the studies of visual backward masking in schizophrenic patients. 
that, unlike the mathematical conception of the present as a point on the temporal line, it is an interval and not a durationless instant; what this Specious Present is meant to capture is our phenomenological present, a duration which is perceived both as present and as temporally extended. In the second section, I have tried to clarify the differences between the various phenomenological presents that different authors may have in mind; in contemporary literature, the most wide-spread interpretation is the phenomenological immediacy associated with our complex perceptions. Finally, I have clarified that what I am proposing here is a model capable of establishing a definite meaning for this phenomenological immediacy. In the third section, I have presented the main models of our temporal phenomenology and showed how the Specious Present appears in them; every model has a story to tell about our phenomenological immediacy and it seems that - if we reject the change of perspective I propose here - we don't have a way to choose. In the fourth section, I have argued that if the difference between the models simply concerns the point at which external data become conscious - where to put the line between memory and present consciousness - there is not much hope for the debate, since it seems to rely on the fallacy of the Cartesian theatre of the mind. In the fifth section, I have proposed the change of perspective which is the object of the present paper: if we intend the dispute between the models as regarding the experiential 'here-now' - much nearer to our phenomenological and practical life - there is the possibility of a new dawn for it. Realizing to having heard two close sounds is something different from realizing to having heard one sound and many seconds later realizing to having heard another sound, one tone higher. Co-consciousness, then, would be defined by the ratio between the temporal distance of the two sounds and our capability to react (in my example, judge them). The rate would define then an extended now, just as in the spatial case I presented the length of our arms and the distance of an object define our extended here. In the sixth and final section, I have stressed the role of anticipation in our phenomenal temporalitysomething which, again, points in the direction of an active role of the window of presentness-, and I considered a possible counterargument: if we don't specify how extended is that window, it could be said, we haven't actually brought about any kind of improvement 
to the debate. I have argued that, even if a lot of experimental work should be done on this topic, my point was merely that the experiments should not be conducted in the belief that we are going to discover the border between present consciousness and memory; instead of focusing on what particular cognitive acts are going on in our mind, the experimenters should focus on our possibility to act and react. My intuition is that the results (the extendedness of the specious present) will vary depending on the particular task set by the experimenters; it is quite natural, after all, to think that the window of our phenomenal presence has a different extension depending on what we are doing or trying to do.

Andrea Roselli

Durham University roselli.uniroma3@gmail.com

\section{References}

Bear, Mark; Connors, Barry; and Paradiso, Michael. 1996. Neuroscience. Exploring the brain. Baltimore: Williams and Wilkins.

Dainton, Barry. 2000. Stream of Consciousness. Unity and Continuity in Conscious Experience. London: Routledge.

Dainton, Barry. 2017. The specious present: further issues. In Stanford Encyclopedia of Philosophy, https://plato.stanford.edu/entries/consciousnesstemporal/specious-present.html

Dainton, Barry. 2017b. Some relevant empirical findings (psychology, psychophysics, neuroscience). In Stanford Encyclopedia of Philosophy, https:// plato.stanford.edu/entries/consciousness-temporal/empirical-findings.html

Herzog, Michael; Roinishvili, Maya; Chkonia, Eka; Brand, Andreas. 2013. Schizophrenia and visual backward masking: a general deficit of target enhancement. Frontiers in Psychology 4: 254.

Hoerl, Christoph. 2013. Husserl, the absolute flow, and temporal experience. Philosophy and Phenomenological Research 86: 376-411.

Hoerl, Christoph. 2014. Time and the domain of consciousness. Annals of the New York Academy of Sciences 1326: 90-6.

James, William. 1890. Principles of Psychology. London: Macmillan.

Lee, Geoffrey. 2014. Extensionalism, atomism, and continuity. In Debates in the Metaphysics of Time, edited by L. Nathan Oaklander, pp. 149-74. London: Bloomsbury Academic.

Le Poidevin, Robert. 2007. The Images of Time: an Essay on Temporal Representation. Oxford: Oxford University Press.

Lockwood, Michael. 2005. The Labyrinth of Time: Introducing the Universe. 
Oxford: Oxford University Press.

Paul, Laurie. 2004. Experience and the arrow. In Chance and Temporal Asymmetry, edited by Alastair Wilson, pp. 175-93. Oxford: Oxford University Press.

Pöppel, Ernst. 1997. The brain's way to create 'nowness'. In Time, Temporality, Now. Experiencing Time and Concepts of Time in an Interdisciplinary Perspective, edited by Harald Atmanspacher and Eva Ruhnau. Berlin: Springer-Verlag.

Prosser, Simon. 2016. Experiencing Time. Oxford: Oxford University Press.

Prosser, Simon. 2017. Rethinking the specious present. In Routledge Handbook of Philosophy of Temporal Experience, edited by Ian Phillips, pp. 146-56. London: Routledge.

Rizzolatti, Giacomo; Gallese, Vittorio; Fogassi, Leonardo; and Fadiga, Luciano. 1996. Premotor cortex and the recognition of motor actions. Cognitive Brain Research 3: 131-41.

Rizzolatti, Giacomo; Gallese, Vittorio; and Fogassi, Leonardo. 2000. Cortical mechanisms subserving object grasping and action recognition: a new view on the cortical motor functions. In The Cognitive Neurosciences, edited by Mauro Gazzaniga, pp. 539-52. Cambridge: MIT Press.

Saccuzzo, Dennis; Cadenhead, Kristin; and Braff, David. 1996. Backward versus forward visual masking deficits in schizophrenic patients: centrally, not peripherally, mediated? American Journal of Psychiatry 153: 1564-70.

Sinigaglia, Corrado. 2008. Enactive understanding and motor intentionality. In Enacting Intersubjectivity: A Cognitive and Social Perspective to Study of Interactions, edited by F. Morganti, A. Carassa and G. Riva, pp. 17-32. Amsterdam: IOS Press.

Sinigaglia, Corrado. 2008b. Mirror neurons: this is the question. Journal of Consciousness Studies 15: 70-92.

Strawson, Galen. 2009. Selves. Oxford: Oxford University Press.

Szelag, Elzbieta. 1997. Temporal integration of the brain as studied with the metronome paradigm. In Time, Temporality, Now. Experiencing Time and Concepts of Time in an Interdisciplinary Perspective, edited by Harald Atmanspacher and Eva Ruhnau. Berlin: Springer-Verlag.

Wittmann, Marc. 2011. Moments in time. Frontiers in Integrative Neuroscience 5: 66. 